Total organic Carbon, extractable organic matter, Rock-Eval parameters, Isoprenoid ratios, Carbon preference index, and isotopic data from cuttings of the AMOCO Cathedral River No. 1 well. 


\begin{tabular}{|c|c|c|c|c|c|c|c|c|c|c|c|c|}
\hline \multirow{3}{*}{$\begin{array}{l}\text { Sample } \\
\text { No. } \\
132109-1\end{array}$} & \multirow{2}{*}{$\begin{array}{l}\text { Depth } \\
\mathrm{ft}\end{array}$} & \multirow[t]{2}{*}{ Formation } & \multirow{2}{*}{$\begin{array}{l}\text { TOC } \\
\%\end{array}$} & \multirow{2}{*}{$\begin{array}{l}\text { EOM } \\
\mathrm{ppm}\end{array}$} & \multirow{2}{*}{$\begin{array}{c}\text { EOM/TOC } \\
\%\end{array}$} & $s_{1}$ & $\mathrm{~S}_{2}$ & $\mathrm{~S}_{3}$ & $\mathrm{HI}$ & OI & \multirow{2}{*}{${ }_{{ }^{\circ} \mathrm{C}}^{\mathrm{T}}$} & \multirow{2}{*}{$R_{0}$} \\
\hline & & & & & & \multicolumn{3}{|c|}{$\mathrm{mg} / \mathrm{g}$ rock } & \multicolumn{2}{|c|}{$\mathrm{mg} / \mathrm{gTOC}$} & & \\
\hline & $5360-5630$ & $\begin{array}{l}\text { Shelikof, } m \text {. } \\
\text { mbr; }\end{array}$ & 0.37 & 274 & 7.4 & 0.09 & 0.54 & 0.40 & 144 & 109 & 431 & 0.7 \\
\hline-2 & $6260-6530$ & $\begin{array}{l}\text { Shelikof, m. } \\
\text { mbr; }\end{array}$ & 0.61 & 406 & 6.6 & 0.12 & 0.71 & 0.46 & 117 & 76 & 435 & 0.7 \\
\hline-3 & $7340-7610$ & $\begin{array}{c}\text { Shelikof, m. } \\
\text { mbr; }\end{array}$ & 0.41 & 475 & 11.6 & 0.08 & 0.44 & 0.58 & 108 & 143 & 428 & 1.1 \\
\hline-4 & $8780-9230$ & $\begin{array}{l}\text { Kialagvik, u. } \\
\text { mbr; }\end{array}$ & 0.85 & 856 & 10.1 & 0.23 & 1.04 & 0.59 & 122 & 70 & 432 & 1.3 \\
\hline-5 & $9950-10,400$ & $\begin{array}{l}\mathrm{Kialagvik,} \mathrm{m} . \\
\mathrm{mbr} ;\end{array}$ & 0.77 & 571 & 7.4 & 0.20 & 0.92 & 0.68 & 119 & 88 & 430 & 1.8 \\
\hline-6 & $11030-11300$ & $\begin{array}{l}\text { Kialagvik, m. } \\
\text { mbr; }\end{array}$ & 0.74 & 317 & 4.3 & 0.18 & 0.48 & 0.52 & 65 & 72 & 432 & 1.8 \\
\hline-7 & $11660-11930$ & $\begin{array}{l}\text { Unnamed, L. } \\
\text { Jur. }\end{array}$ & 0.81 & 284 & 3.5 & 0.18 & 0.37 & 0.38 & 45 & 48 & 433 & 1.9 \\
\hline-8 & $12020-12110$ & $\begin{array}{c}\text { Unnamed, L. } \\
\text { Jur. }\end{array}$ & 1.07 & 1134 & 10.6 & 0.32 & 1.29 & 0.82 & 121 & 76 & 425 & 2.0 \\
\hline-9 & $13820-14090$ & $\begin{array}{l}\text { Unnamed, L. } \\
\text { Jur. }\end{array}$ & 1.07 & 295 & 2.8 & 0.22 & 0.51 & 0.46 & 48 & 43 & 428 & 2.4 \\
\hline-10 & $14090-1436$ & $\begin{array}{c}\text { Unnamed, U. } \\
\text { Triassic }\end{array}$ & 0.74 & 405 & 5.5 & 0.14 & 0.58 & 0.50 & 78 & 68 & 429 & 2.4 \\
\hline
\end{tabular}


Table 2. Bulk composition, isoprenoid ratios, carbon preference index, and isotopic data obtained from composite cuttings from the Amoco Cathedral River \#1 well.

$\begin{array}{rccccr}\begin{array}{c}\text { Sample } \\ \text { No. }\end{array} & \begin{array}{c}\text { Depth } \\ \mathrm{ft}\end{array} & \operatorname{Pr} / \mathrm{Cl} & \mathrm{Ph} / \mathrm{C18} & \mathrm{Pr} / \mathrm{Ph} & \mathrm{CPI} \\ 132109-1 & 5360-5630 & 0.48 & 0.38 & 1.76 & 1.07 \\ -2 & 6260-6530 & 0.48 & 0.31 & 2.09 & 1.14 \\ -3 & 7340-7610 & 0.47 & 0.36 & 1.44 & 1.00 \\ -4 & 8780-9230 & 0.50 & 0.31 & 2.08 & 1.01 \\ -5 & 9950-10,400 & 0.45 & 0.31 & 2.11 & 1.11 \\ -6 & 11030-11300 & 0.43 & 0.31 & 2.15 & 1.01 \\ -7 & 11660-11930 & 0.45 & 0.31 & 2.29 & 1.09 \\ -8 & 12020-12110 & 0.51 & 0.32 & 2.01 & 1.03 \\ -9 & 13820-14090 & 0.51 & 0.38 & 2.17 & 0.87 \\ -10 & 14090-1436 & 0.48 & 0.36 & 2.05 & 0.90\end{array}$


Table 1

FILE NO. 85111

Mobil Geochemistry Job No. 1321-9

ROCK-EVAL PYROLYSIS

\begin{tabular}{|c|c|c|c|c|c|c|c|c|c|c|}
\hline $\begin{array}{l}\text { Sample } \\
\text { Number }\end{array}$ & TOC & S1 & $\mathrm{Gm}_{\mathrm{S} 2} \mathrm{RO}_{\mathrm{O}}$ & S3 & $\begin{array}{c}\text { Hydrogen } \\
\text { Index }\end{array}$ & $\begin{array}{l}\text { Oxygen } \\
\text { Index }\end{array}$ & $\begin{array}{r}\text { oil } \\
\text { Shows }\end{array}$ & $\begin{array}{l}\text { or Gas } \\
\text { Potential }\end{array}$ & $\begin{array}{l}\text { Trans } \\
\text { Ratio }\end{array}$ & $\begin{array}{l}\text { TMAX } \\
(\operatorname{Deg} C)\end{array}$ \\
\hline 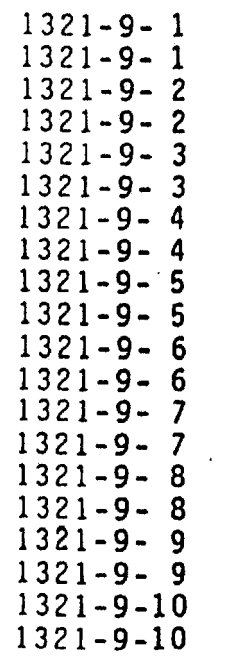 & $\begin{array}{l}0.39 \\
0.35 \\
0.64 \\
0.58 \\
0.41 \\
0.40 \\
0.87 \\
0.83 \\
0.80 \\
0.74 \\
0.73 \\
0.74 \\
0.83 \\
0.79 \\
1.07 \\
1.06 \\
1.06 \\
1.07 \\
0.74 \\
0.74\end{array}$ & $\begin{array}{l}0.09 \\
0.09 \\
0.11 \\
0.12 \\
0.08 \\
0.09 \\
0.23 \\
0.23 \\
0.20 \\
0.20 \\
0.18 \\
0.18 \\
0.18 \\
0.18 \\
0.28 \\
0.36 \\
0.21 \\
0.23 \\
0.13 \\
0.16\end{array}$ & $\begin{array}{l}0.58 \\
0.49 \\
0.68 \\
0.74 \\
0.44 \\
0.44 \\
1.05 \\
1.03 \\
0.92 \\
0.91 \\
0.50 \\
0.47 \\
0.37 \\
0.37 \\
1.28 \\
1.30 \\
0.52 \\
0.50 \\
0.58 \\
0.58\end{array}$ & $\begin{array}{l}0.37 \\
0.43 \\
0.43 \\
0.49 \\
0.66 \\
0.50 \\
0.53 \\
0.66 \\
0.74 \\
0.62 \\
0.57 \\
0.47 \\
0.42 \\
0.35 \\
0.83 \\
0.80 \\
0.47 \\
0.45 \\
0.53 \\
0.48\end{array}$ & $\begin{array}{r}148.7 \\
140.0 \\
106.3 \\
127.6 \\
107.3 \\
110.0 \\
120.7 \\
124.1 \\
115.0 \\
123.0 \\
68.5 \\
63.5 \\
44.6 \\
46.8 \\
119.6 \\
122.6 \\
49.1 \\
46.7 \\
78.4 \\
78.4\end{array}$ & $\begin{array}{r}94.9 \\
122.9 \\
67.2 \\
84.5 \\
161.0 \\
125.0 \\
60.9 \\
79.5 \\
92.5 \\
83.8 \\
78.1 \\
63.5 \\
50.6 \\
44.3 \\
77.6 \\
75.5 \\
44.3 \\
42.1 \\
71.6 \\
64.9\end{array}$ & $\begin{array}{l}0.09 \\
0.09 \\
0.11 \\
0.12 \\
0.08 \\
0.09 \\
0.23 \\
0.23 \\
0.20 \\
0.20 \\
0.18 \\
0.18 \\
0.18 \\
0.18 \\
0.28 \\
0.36 \\
0.21 \\
0.23 \\
0.13 \\
0.16\end{array}$ & $\begin{array}{l}0.67 \\
0.58 \\
0.79 \\
0.86 \\
0.52 \\
0.53 \\
1.28 \\
1.26 \\
1.12 \\
1.11 \\
0.68 \\
0.65 \\
0.55 \\
0.55 \\
1.56 \\
1.66 \\
0.73 \\
0.73 \\
0.71 \\
0.74\end{array}$ & $\begin{array}{l}0.13 \\
0.16 \\
0.14 \\
0.14 \\
0.15 \\
0.17 \\
0.18 \\
0.18 \\
0.18 \\
0.18 \\
0.26 \\
0.28 \\
0.33 \\
0.33 \\
0.18 \\
0.22 \\
0.29 \\
0.32 \\
0.18 \\
0.22\end{array}$ & $\begin{array}{l}431 \\
431 \\
436 \\
434 \\
428 \\
428 \\
432 \\
432 \\
432 \\
428 \\
432 \\
431 \\
433 \\
433 \\
426 \\
423 \\
428 \\
427 \\
431 \\
427\end{array}$ \\
\hline
\end{tabular}

TOC = Total Organic Carbon

Hydrogen Index $=($ S2/TOC $) \times 100$
$0 x y g e n$ Index $=(S 3 / T O C) \times 100$

0 il or Gas Shows = SI 0il or Gas Potential = S1+S2

Transformation Ratio $=S 1 /(S 1+S 2)$ 


$\begin{array}{lll}\text { SAMPLE } & 1321-9-1 & \text { FILE'NO } 1321-9 \\ \text { LOCATION } & \text { ALASKA } & \text { SAMP'ID } 85 / 01541 \\ \text { DEPTH } & 5360-5630 \mathrm{FT} & \text { SAMPTYPE CUTTINGS } \\ \text { GLC'F } & \text { RA2564 } & \\ & \text { ALKANE SUMMARY DATA } & \end{array}$

RATIO PRISTANE/PHYTANE 1.76

RATIO PRISTANE/NEIT

RATIO PHYTANE/NCI8
.48

.38
CARBON PREFERENCE INDEX 1.07

\section{$x$ of Total}

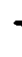

NC10

NC1 1

NC12

NC13

NC14

NC15

NC16

NC17

NC18

NC19

NC2 0

NC2 1

NC22

$\mathrm{NC} 23$
1.432

1.464

.574

.907

2.065

3.832

4.961

5.358

3.796

2.798

1.822

1.562

1.500

1.437

\section{Isoprenoids}

C15 IP

C16IP

C18IP

PRISTANE

PHYTANE
.401

1.068

1.658

2.560

1.457

\section{x of Total}

$\begin{array}{lr}N C 24 & 1.327 \\ N C 25 & 1.128 \\ N C 26 & .883 \\ N C 27 & .714 \\ N C 28 & .590 \\ N C 29 & .607 \\ N C 30 & .422 \\ N C 31 & .327 \\ N C 32 & .236 \\ N C 33 & .174 \\ N C 34 & .148 \\ N C 35 & .064 \\ N C 36 & .017\end{array}$




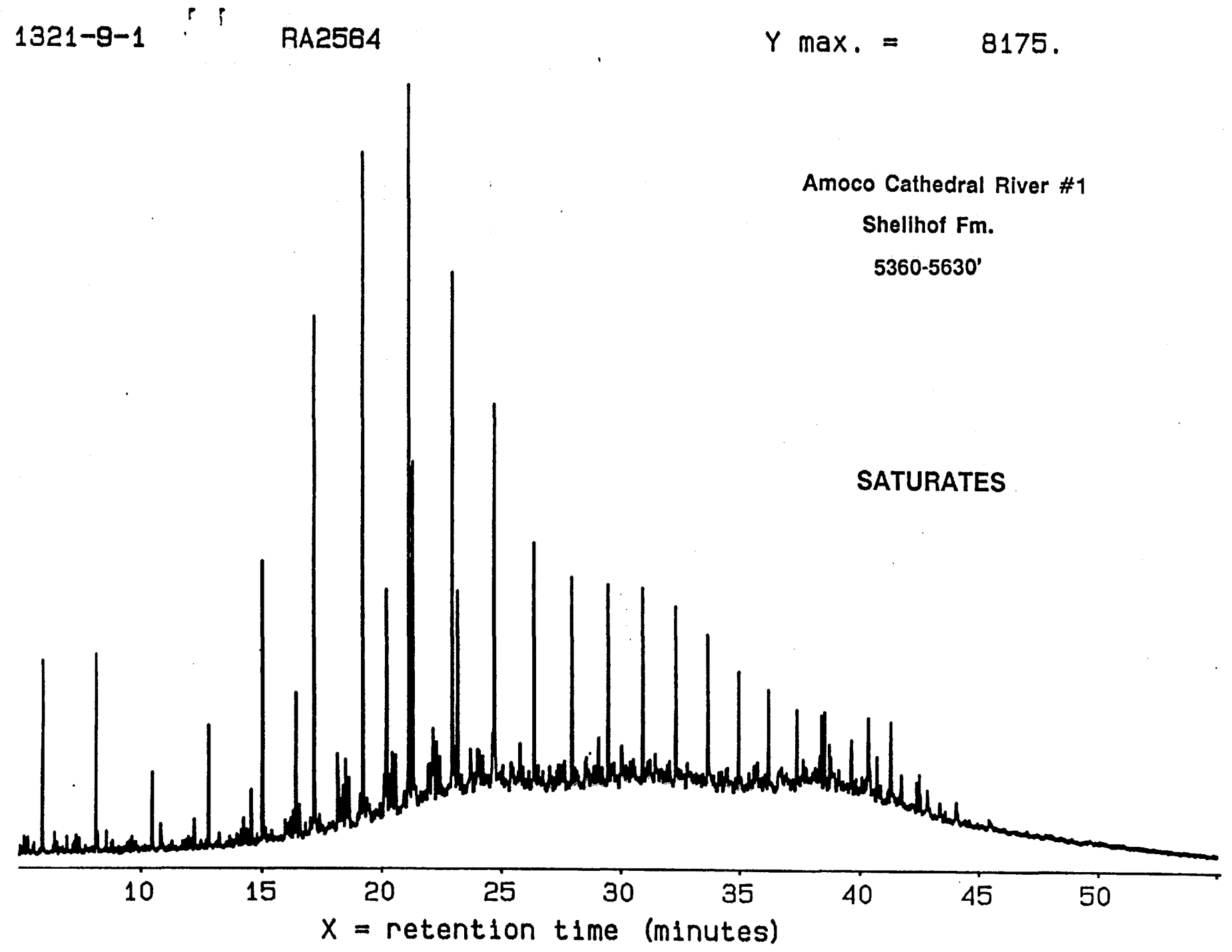




\begin{tabular}{lll} 
SAMPLE & $1321-9-2$ & FILE'NO $1321-9$ \\
LOCATION & ALASKA & SAMP'ID B5/01542 \\
DEPTH & 6260-6530 FT & SAMPTYPE CUTTINGS \\
GLC'F & RA2565 & \\
\hline & ALKANE SUMMARY DATA &
\end{tabular}

\section{RATID PRISTANE/PHYTANE 2.09 RATIO PRISTANE/NCIT \\ RATIO PHYTANE/NC18}

\section{CARBON PREFERENCE INDEX 1.14}

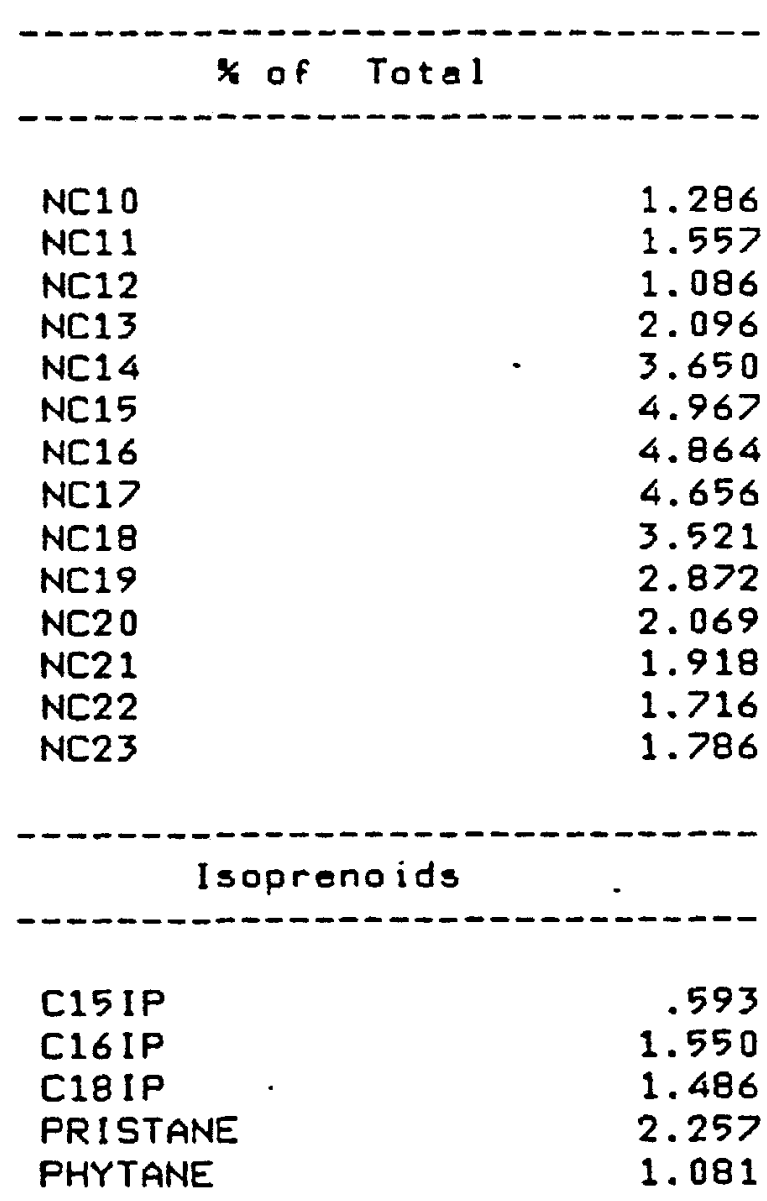




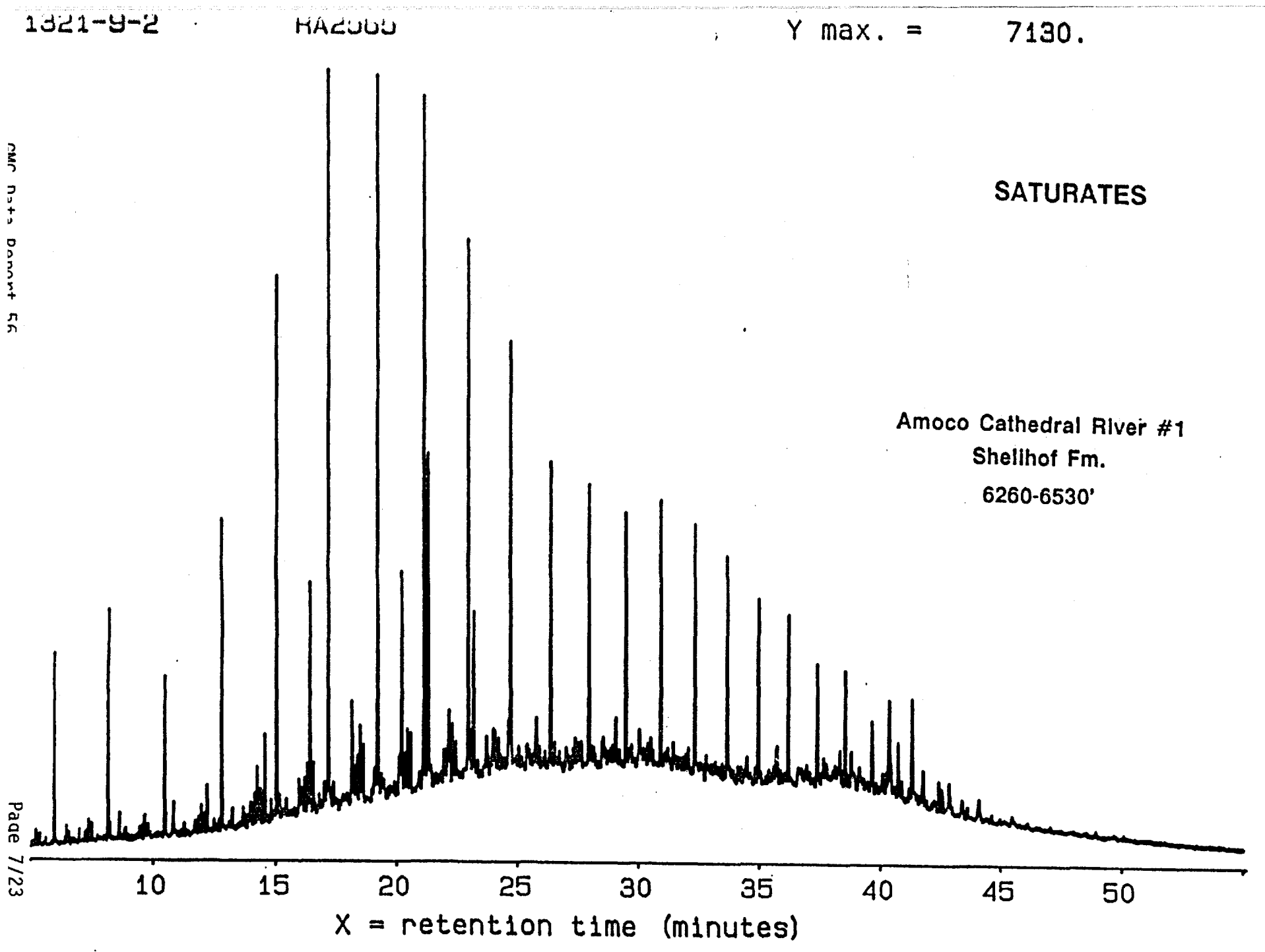




\begin{tabular}{lll} 
SAMPLE & $1321-9-3$ & FILE'NO $1321-9$ \\
LOCATION & ALASKA & SAMP'ID $85 / 01543$ \\
DEPTH & $7340-7610 \mathrm{FT}$ & SAMPTYPE CUTTINGS \\
GLC'F & RA2566 & \\
\hline & ALKANE SUMMARY DATA &
\end{tabular}

RATIO PRISTANE/PHYTANE 1.44 RATIO PRISTANE/NCIT

RAT IO PHYTANE/NC18 .47

.36
CARBON PREFERENCE INDEX 1.00

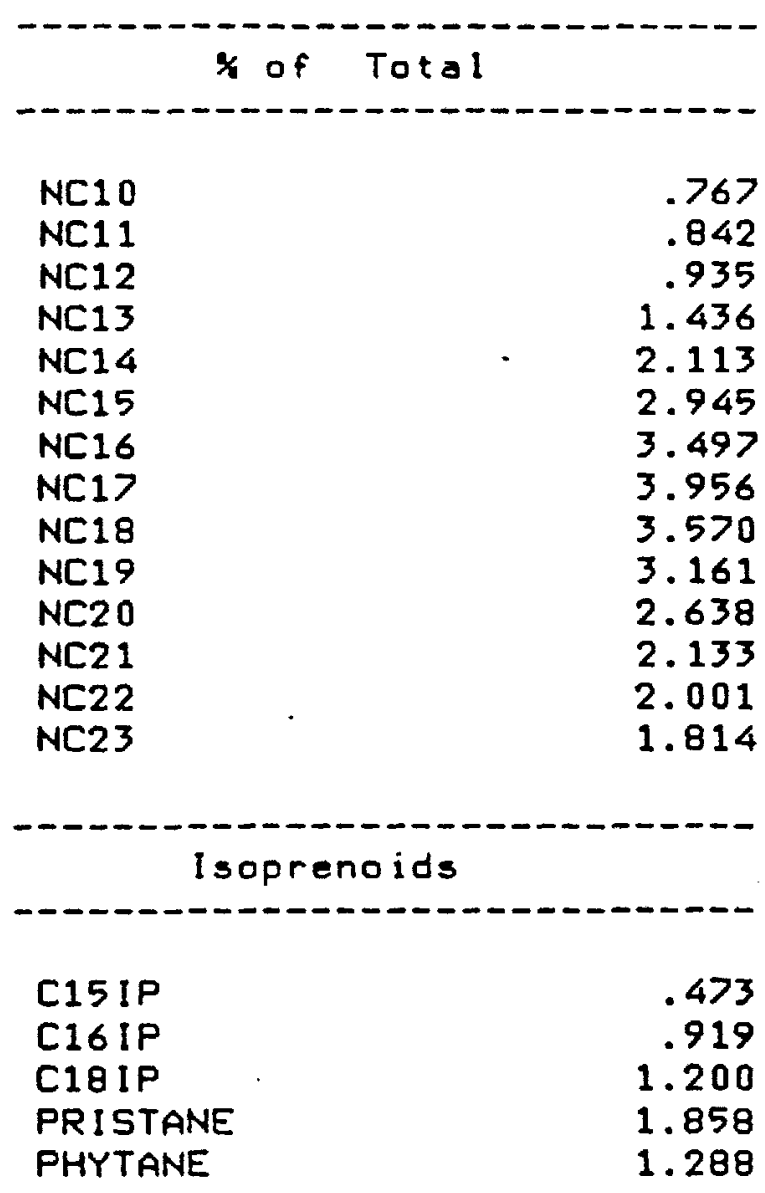


$Y \max .=8920$.

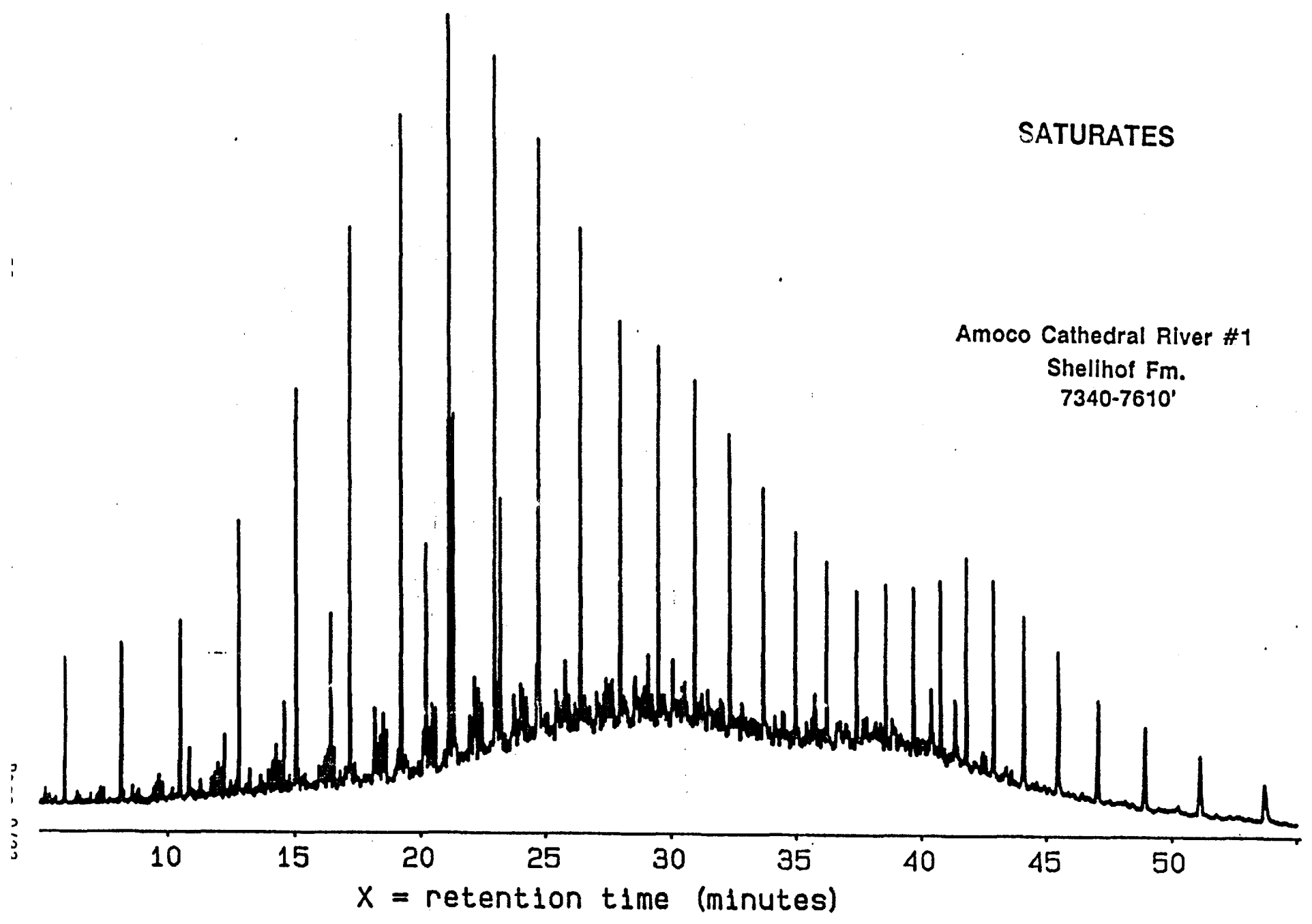




$\begin{array}{ll}\text { SAMPLE } & 1321-9-4 \\ \text { LOCATION } & \text { ALASKA } \\ \text { DEPTH } & 8>80-9230 \mathrm{FT} \\ \text { GLC'F } & \text { RA2567 }\end{array}$

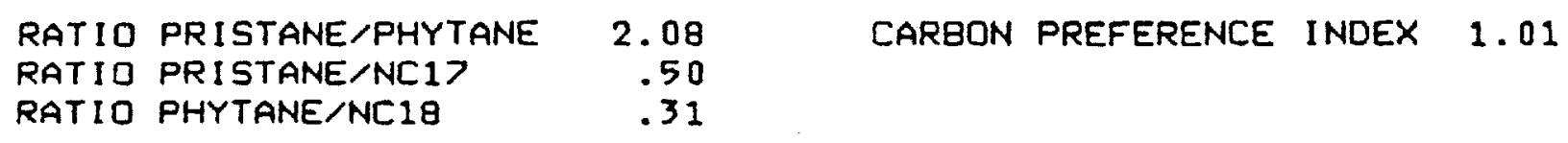

\section{* of Total}

\section{NC10}

NC11

NC12

NC13

NC14

NC15

NC16

NC17

NC18

NC19

NC2 0

NC2 1

NC22

NC23
.390

.666

.680

1.415

3.152

5.132

6.094

6.420

5.047

4.122

2.916

1.914

1.304

1.000

I soprenoids

C15IP

.333

C16IP

1.192

C18IP

2.146

PRISTANE

3.210

PHYTANE

1.543

\section{* of Total}

$\begin{array}{ll}N C 24 & .695 \\ N C 25 & .578 \\ N C 26 & .504 \\ N C 27 & .462 \\ N C 28 & .394 \\ N C 29 & .479 \\ N C 30 & .454 \\ N C 31 & .392 \\ N C 32 & .392 \\ N C 33 & .280 \\ N C 34 & .200 \\ N C 35 & .080 \\ N C 36 & .005\end{array}$




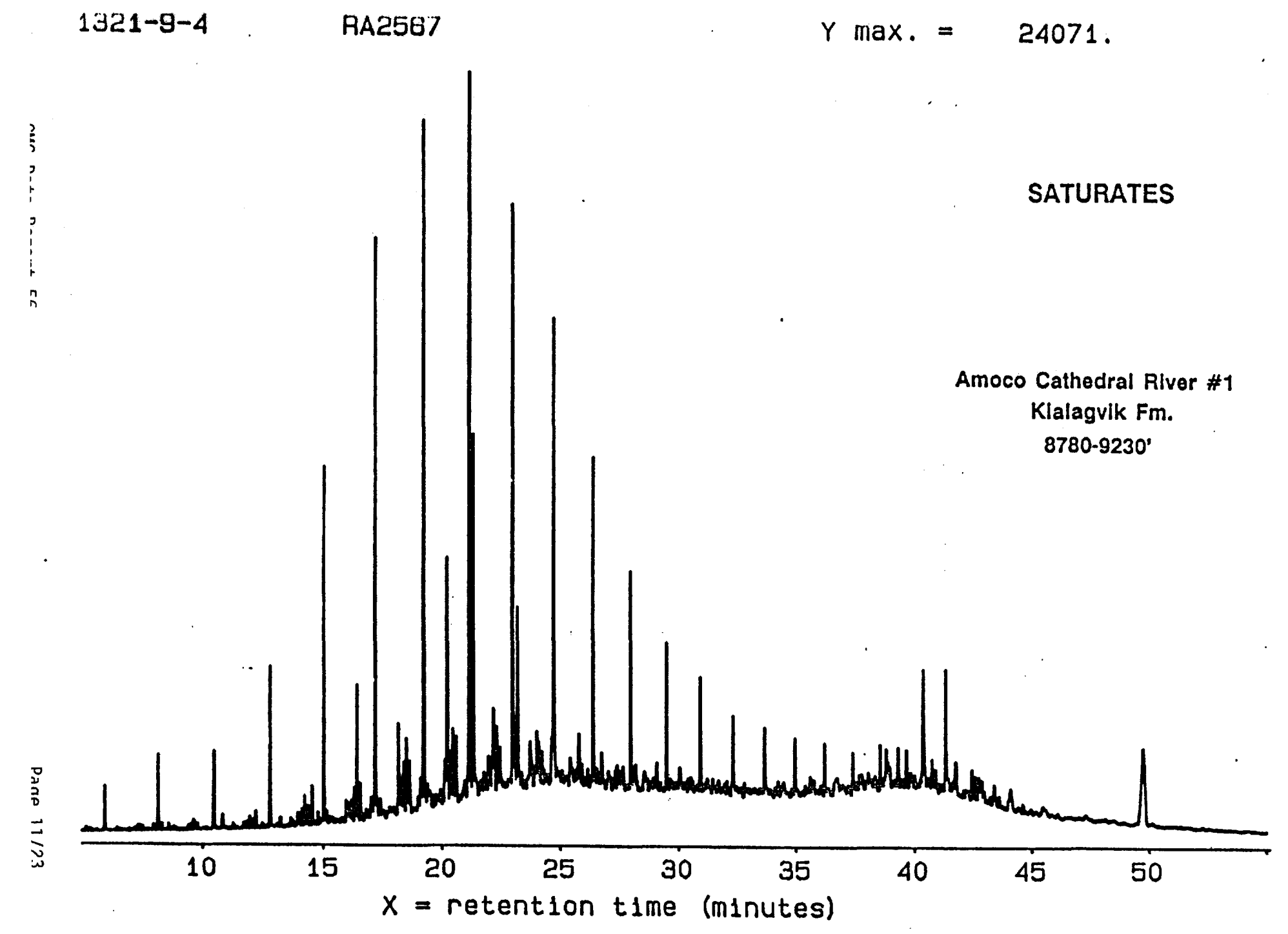




$\begin{array}{ll}\text { SAMPLE } & 1321-9-5 \\ \text { LOCATION } & \text { ALASKA } \\ \text { DEPTH } & 9950-10400 \text { FT } \\ \text { GLC'F } & \text { RA2568 }\end{array}$

$--\cdots--\cdots-\cdots$

ALKANE SUMMARY DATA

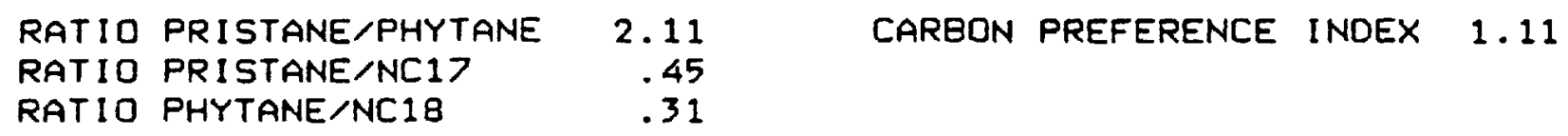

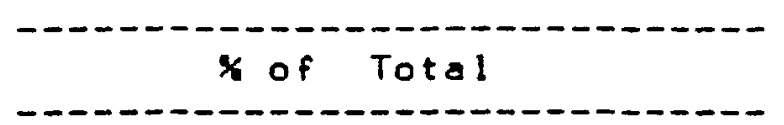

NC10

NC11

NC12

$\mathrm{NC} 13$

NC14

NC15

NC16

NC17

NC18

NC19

NC2 0

NC2 1

NC22

NC23
.828

1.163

1.263

2.703

4.879

6.370

6.255

5.211

3.572

2.601

2.082

1.688

1.529

1. $3>1$

\section{x of Total}

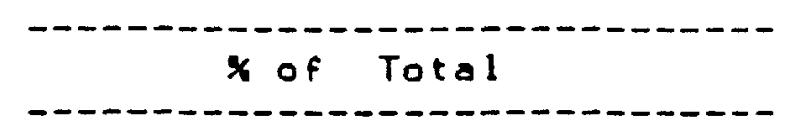

NC24

1.188

NC25

1.008

NC26

.783

NC27

.626

NC2B

.452

NC29

.390

NC30

NC3 1

.234

NC32

.165

.110

NC33

.081

NC34

.053

NC35

.025

NC36

Isoprenoids

C15IP

C16IP

C18IP

PRISTANE

PHYTANE
.774

1.961

2.056

2.348

1.113 
$y \max .=16 y 26$.

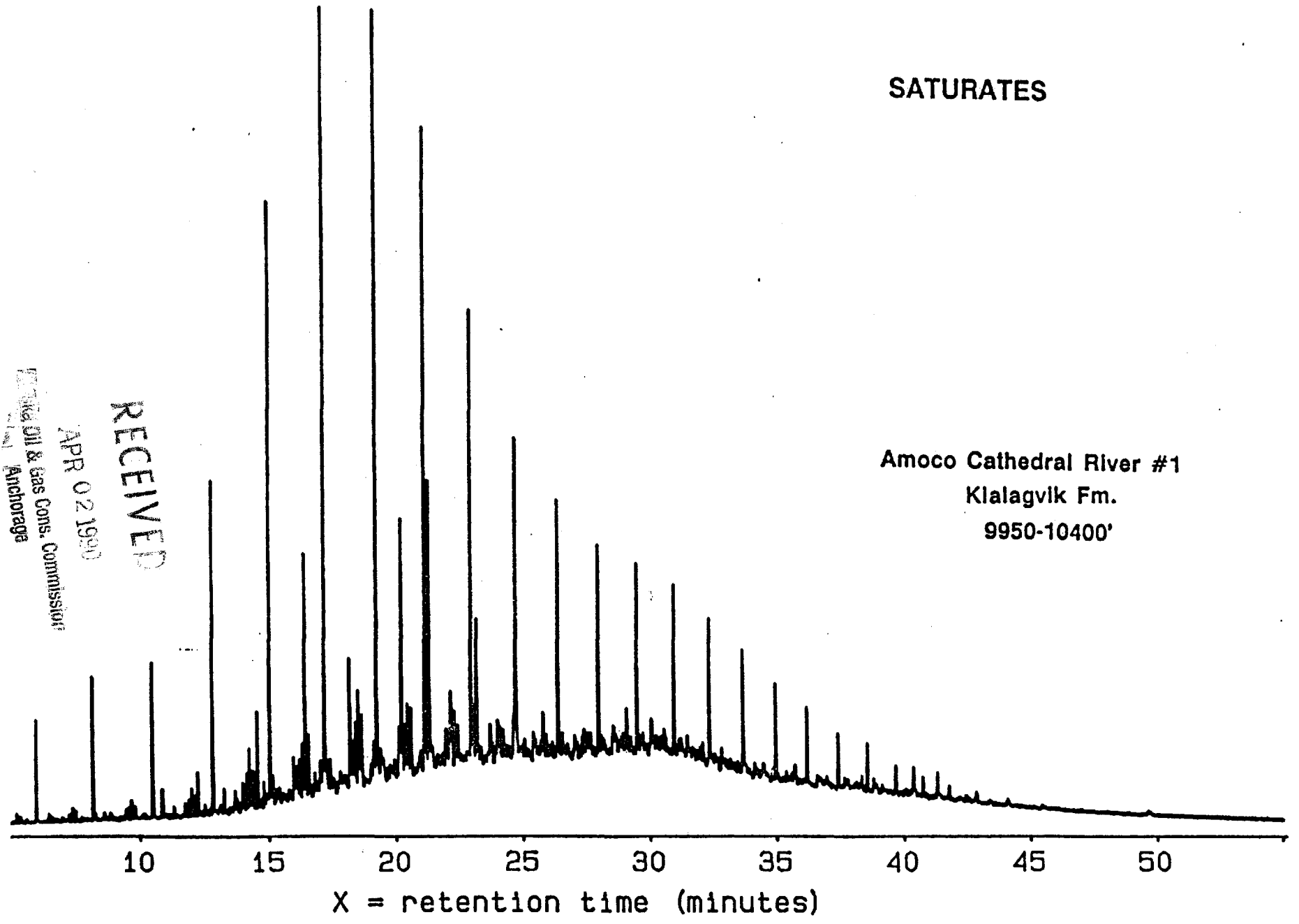




$\begin{array}{lll}\text { SAMPLE } & 1321-9-6 & \text { FILE'NO } 1321-9 \\ \text { LOCATION } & \text { ALASKA } & \text { SAMPIID B5/01546 } \\ \text { OEPTH } & 11030-11300 \mathrm{FT} & \text { SAMPTYPE CUTTINGS } \\ \text { GLC'F } & \text { RA2569 } & \end{array}$

$\begin{array}{lrrr}\text { RATIO PRISTANE/PHYTANE } & 2.15 & \text { CARBON PREFERENCE INDEX } & 1.01 \\ \text { RATIO PRISTANE/NCIT } & .43 & & \\ \text { RATIO PHYTANE/NCIB } & .31 & \end{array}$

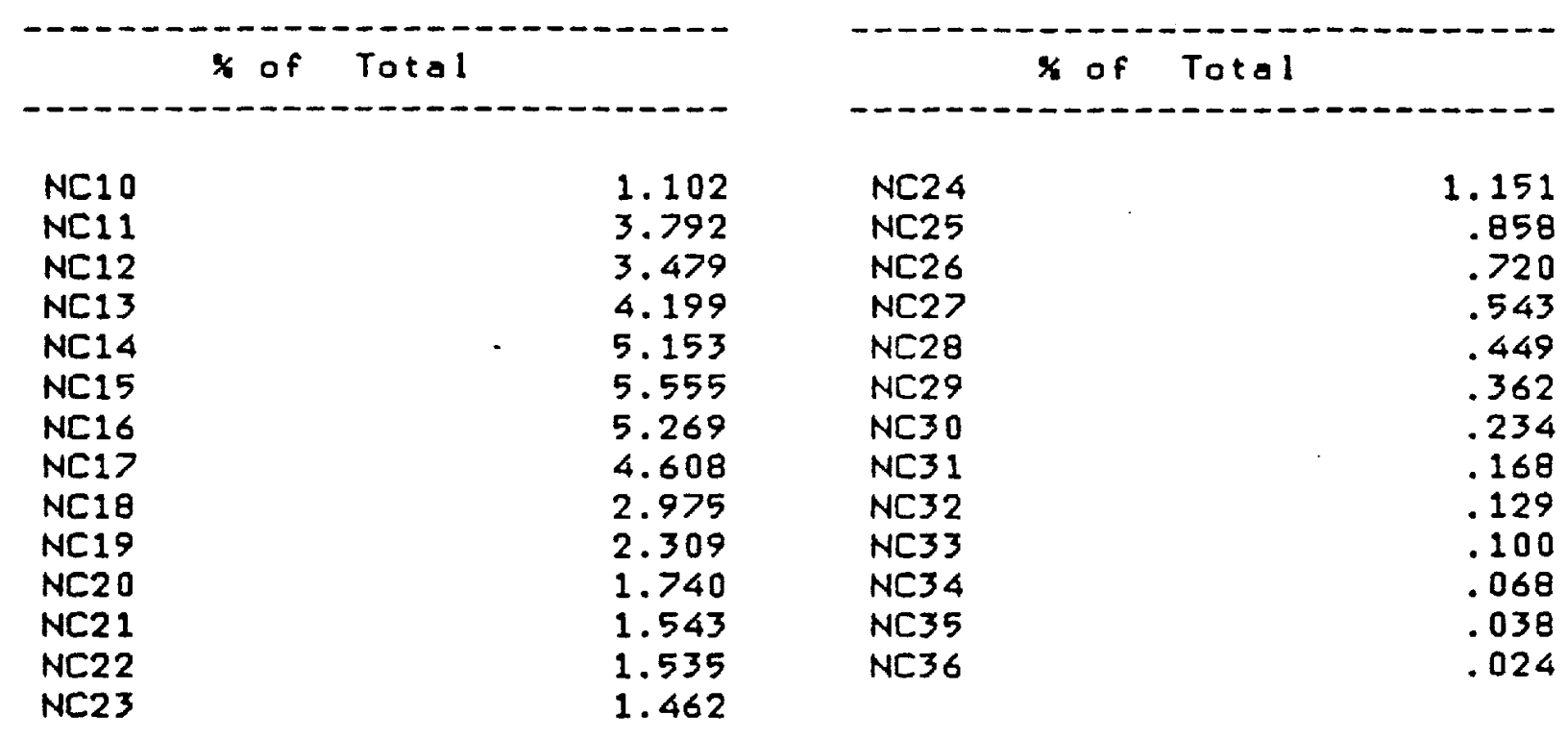

Isoprenoids

C15IP

.917

C16IP

1.840

C18IP

1.761

PRISTANE

1.977

PHYTANE 
$1 J 21-9-6$ 'AA2569

$Y \max .=20170$.

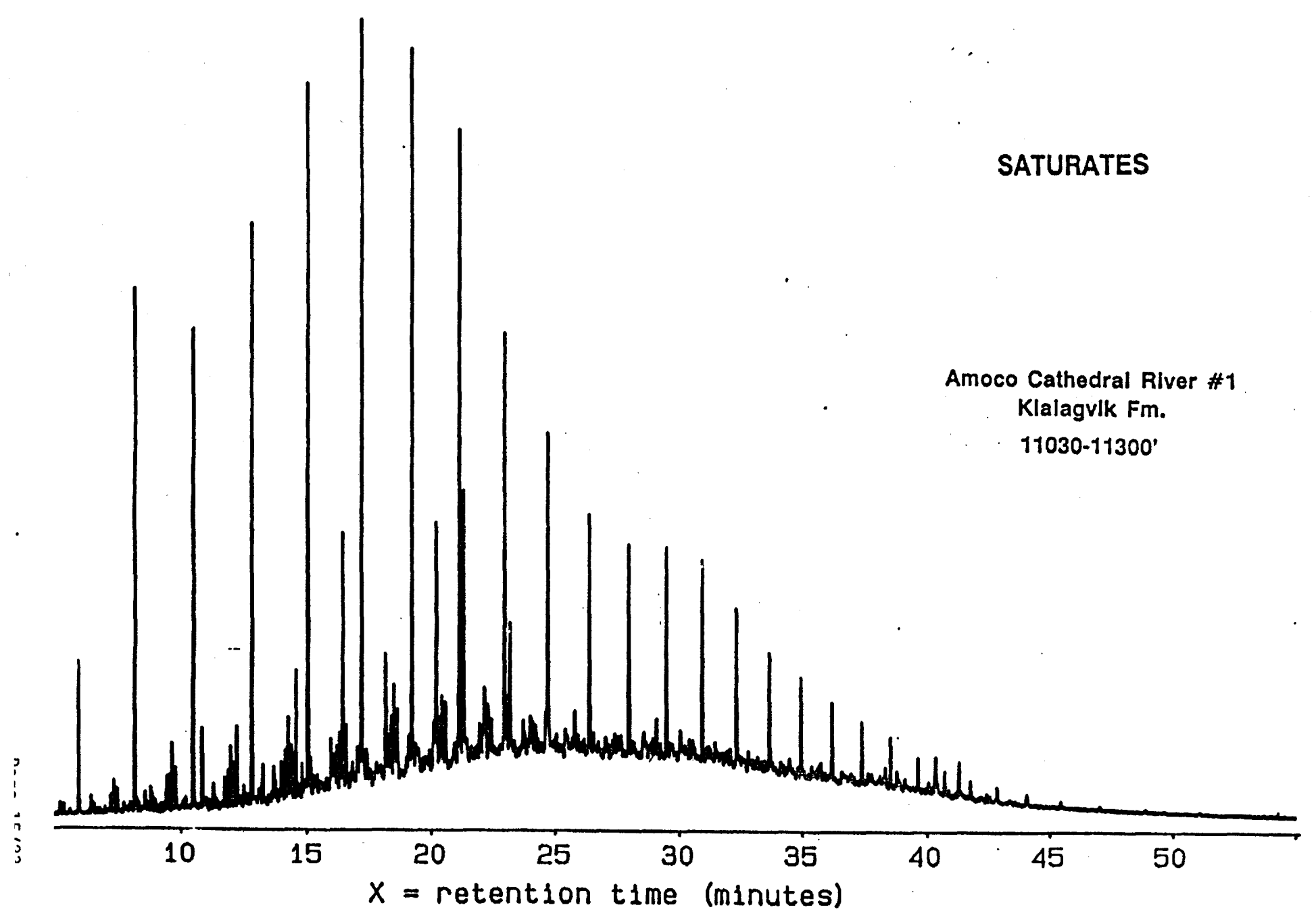




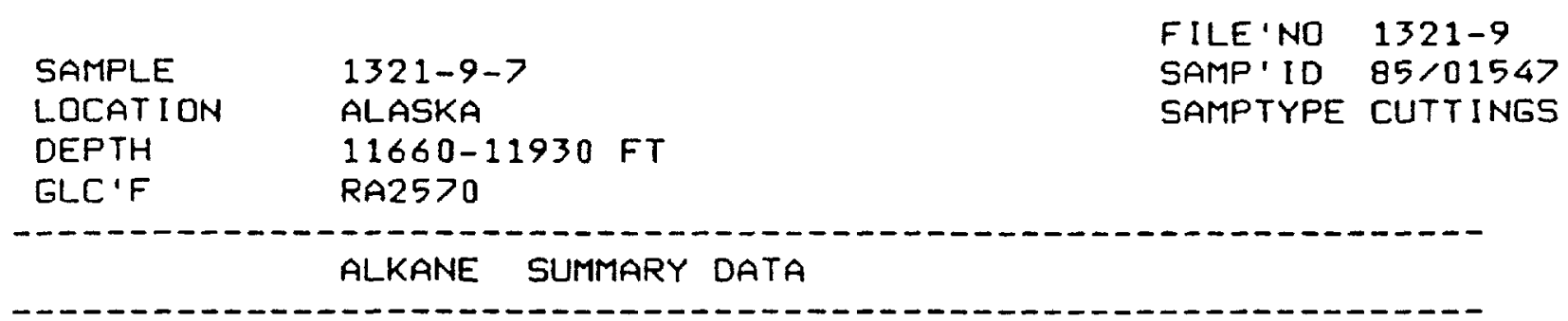

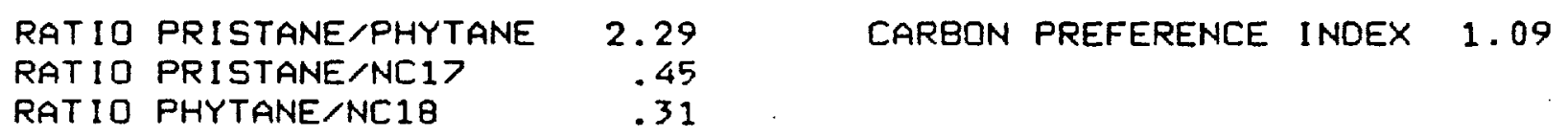

* of Total

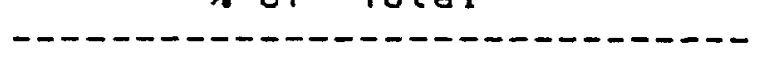

\section{NC10}

NC11

NC12

NC13

NC14

NC15

NC16

NC17

NC1日

NC19

NC20

NC2 1

NC22

NC23
.840

2.803

3.233

4.430

6.225

6.820

6.231

5.219

3.252

2.205

1.551

1.199

1.091

1.145

\section{* of Total}

- - - - - $x$ of

NC24

1.013

NC25

.818

NC26

NC27

.596

.433

NC28

.320

NC29

.257

$N C 30$

NC3 1

NC32

.161

.098

.064

$\mathrm{NC} 33$

.049

NC34

NC35

.033

.009

NC36

Isoprenoids

C15IP

C16IP

C18 IP

PRISTANE

PHYTANE
1.002

2.303

2.130

2.334

1.019 


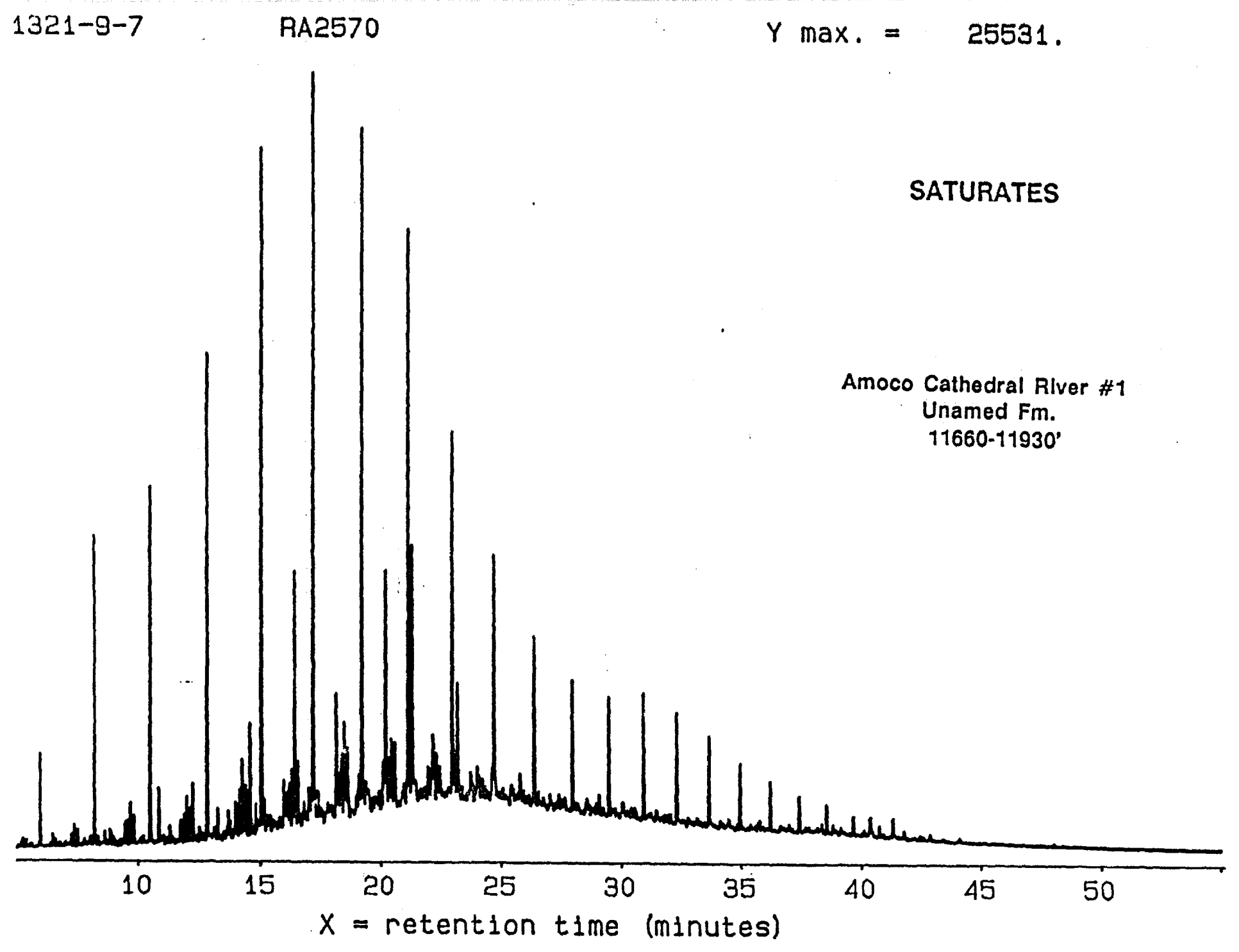




$\begin{array}{lll}\text { SAMPLE } & 1321-9-8 & \text { FILE'NO } 1321-9 \\ \text { LOCATION } & \text { ALASKA } & \text { SAMP'ID } 85 / 01549 \\ \text { DEPTH } & 12020-12470 \text { FT } & \text { SAMPTYPE CUTTINGS } \\ \text { GLC'F } & \text { RA2571 } & \\ & \text { ALKANE SUMMARY DATA } & \end{array}$

$\begin{array}{lrrr}\text { RATIO PRISTANE/PHYTANE } & 2.01 & \text { CARBON PREFERENCE INDEX } & 1.03 \\ \text { RATIO PRISTANE/NCI7 } & .51 & & \\ \text { RATIO PHYTANE/NCI8 } & .32 & \end{array}$

\begin{tabular}{|c|c|c|c|}
\hline NC10 & .017 & NC24 & 1.531 \\
\hline NC11 & .060 & NC25 & 1.278 \\
\hline NC12 & .146 & NC26 & 1.076 \\
\hline $\mathrm{NC}_{13}$ & .728 & NC27 & .862 \\
\hline $\mathrm{NC} 14$ & 2.560 & NC28 & .648 \\
\hline NC15 & $5.1>2$ & NC29 & .547 \\
\hline NC16 & 6.270 & NC3O & .401 \\
\hline NC17 & 6.866 & NC31 & .286 \\
\hline NC18 & 5.422 & NC32 & .294 \\
\hline NC19 & 4.775 & NC33 & .203 \\
\hline NC2 0 & 3.651 & NC34 & .127 \\
\hline NC2 1 & 2.758 & NC35 & .018 \\
\hline $\begin{array}{l}\mathrm{NC} 22 \\
\mathrm{NC} 23\end{array}$ & 2.181 & NC36 & .015 \\
\hline
\end{tabular}

Isoprenoids

$\begin{array}{lr}\text { C15IP } & .326 \\ \text { C16IP } & 1.281 \\ \text { C18IP } & 2.420 \\ \text { PRISTANE } & 3.529 \\ \text { PHYTANE } & 1.756\end{array}$


$Y \max .=16546$.

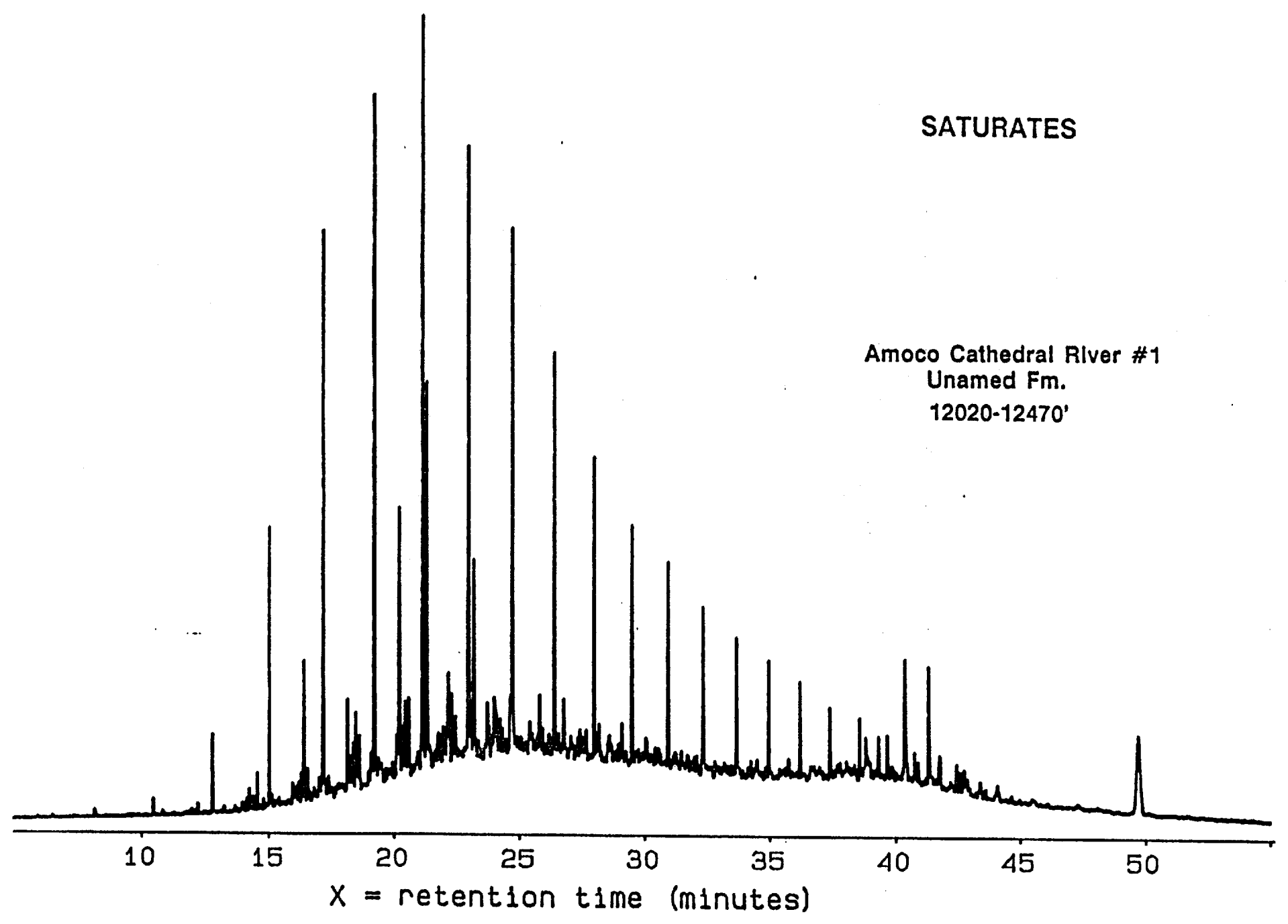




$\begin{array}{lll}\text { SAMPLE } & 1321-9-9 & \text { FILE'NO } 1321-9 \\ \text { LOCATION } & \text { ALASKA } & \text { SAMP'IO } 85 / 01549 \\ \text { DEPTH } & 13820-14090 \mathrm{FT} & \text { SAMPTYPE CUTTINGS } \\ \text { GLC'F } & \text { RA2572 } & \\ & \text { ALKANE SUMMARY DATA } & \end{array}$

RATIO PRISTANE/PHYTANE 2.17 RATIO PRISTANE/NCID RATIO PHYTANE/NC18
17
.51

.38

\section{CARBON PREFERENCE INDEX .87}

\section{* of Total}

- - - -

NC10

NC1 1

NC12

NC13

NC14

NC15

NC16

NC17

NC18

NC19

NC20

NC2 1

NC2 2

$\mathrm{NC} 23$
.772

4.045

5.714

6.761

7.348

6.484

5.323

3.258

2.042

1.194

.792

.730

.538
4.108

\section{* of Total}

NC2 4

NC25

NC26

NC27

NC28

NC29

NC30

NC31

NC32

NC33

NC34

NC35

NC36
.291

.178

.130

.110

.096

.077

.106

.066

.112

.084

.055

.022

.003

I soprenoids

C15 IP

.972

C16IP

2.302

C18IP

2.334

PRISTANE

2.690

PHYTANE

1.240 


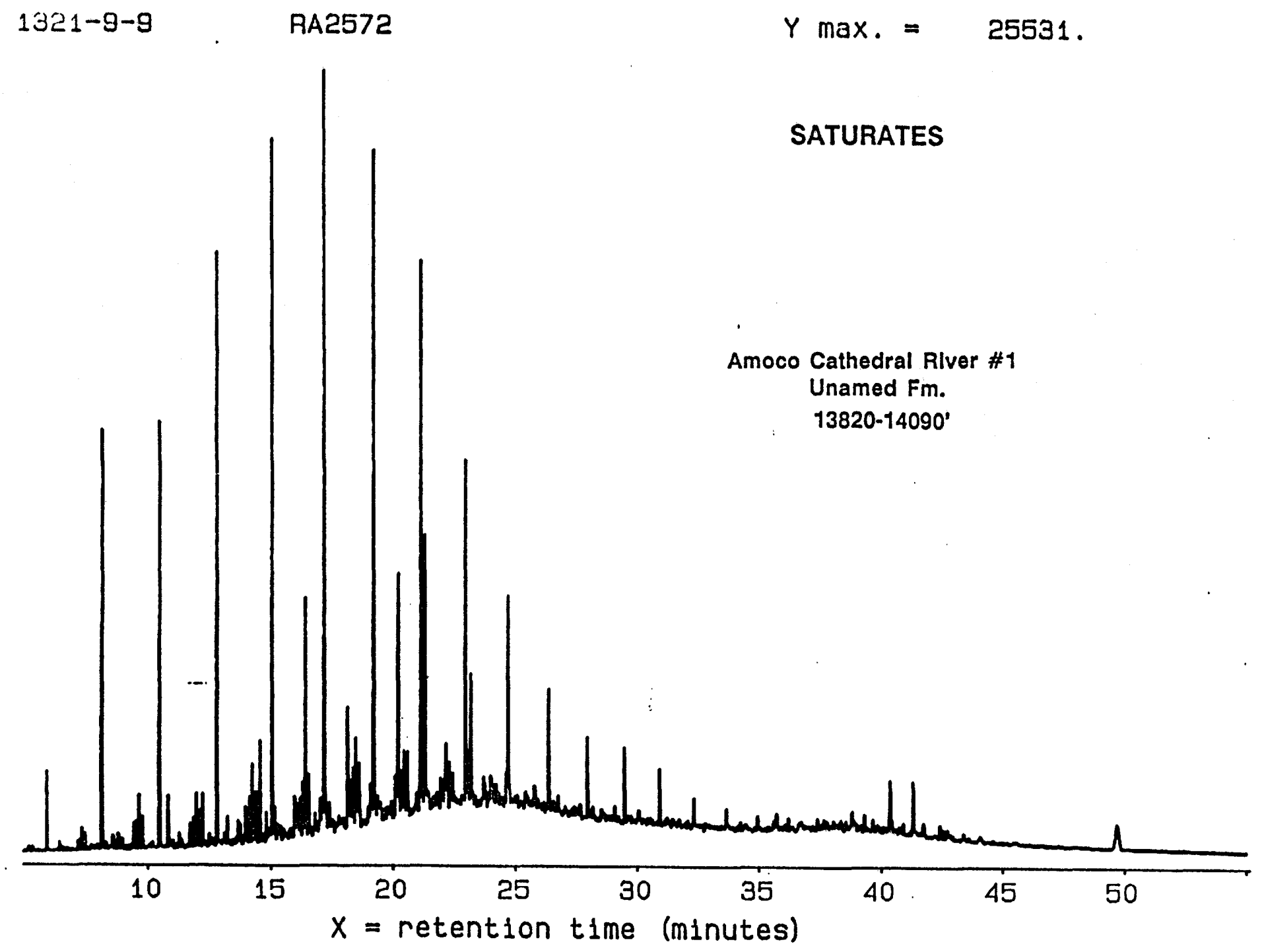




$\begin{array}{lll}\text { SAMPLE } & 1321-9-10 & \text { FILE'NO } 1321-9 \\ \text { LOCATION } & \text { ALASKA } & \text { SAMP'ID B5/01550 } \\ \text { OEPTH } & 14090-14360 \mathrm{FT} & \text { SAMPTYPE CUTTINGS } \\ \text { GLC'F } & \text { RA2573 } & \end{array}$

RATID PRISTANE/PHYTANE 2.05

RATIO PRISTANE/NCI) . 78

RATIO PHYTANE/NC18
CARBON PREFERENCE INDEX .90

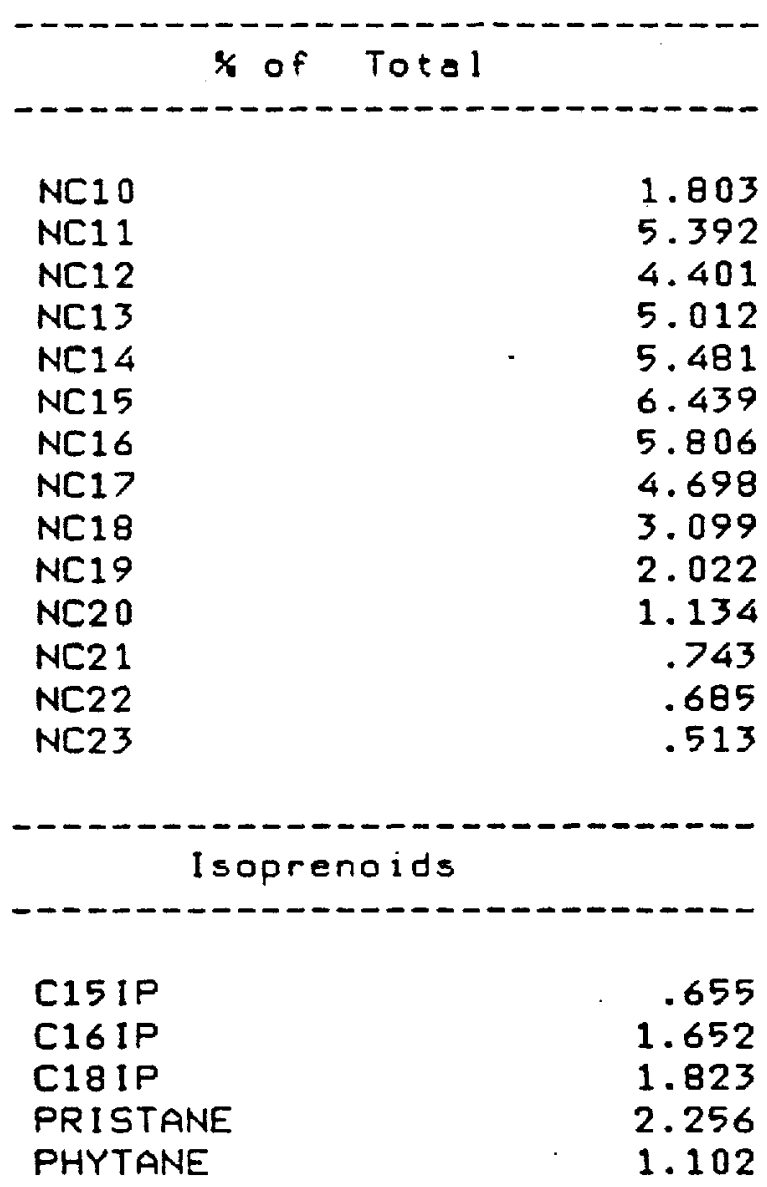

.298

.210

.134

.071

.095

.098

.135

.112

.165

.131

.069

.010

.007 


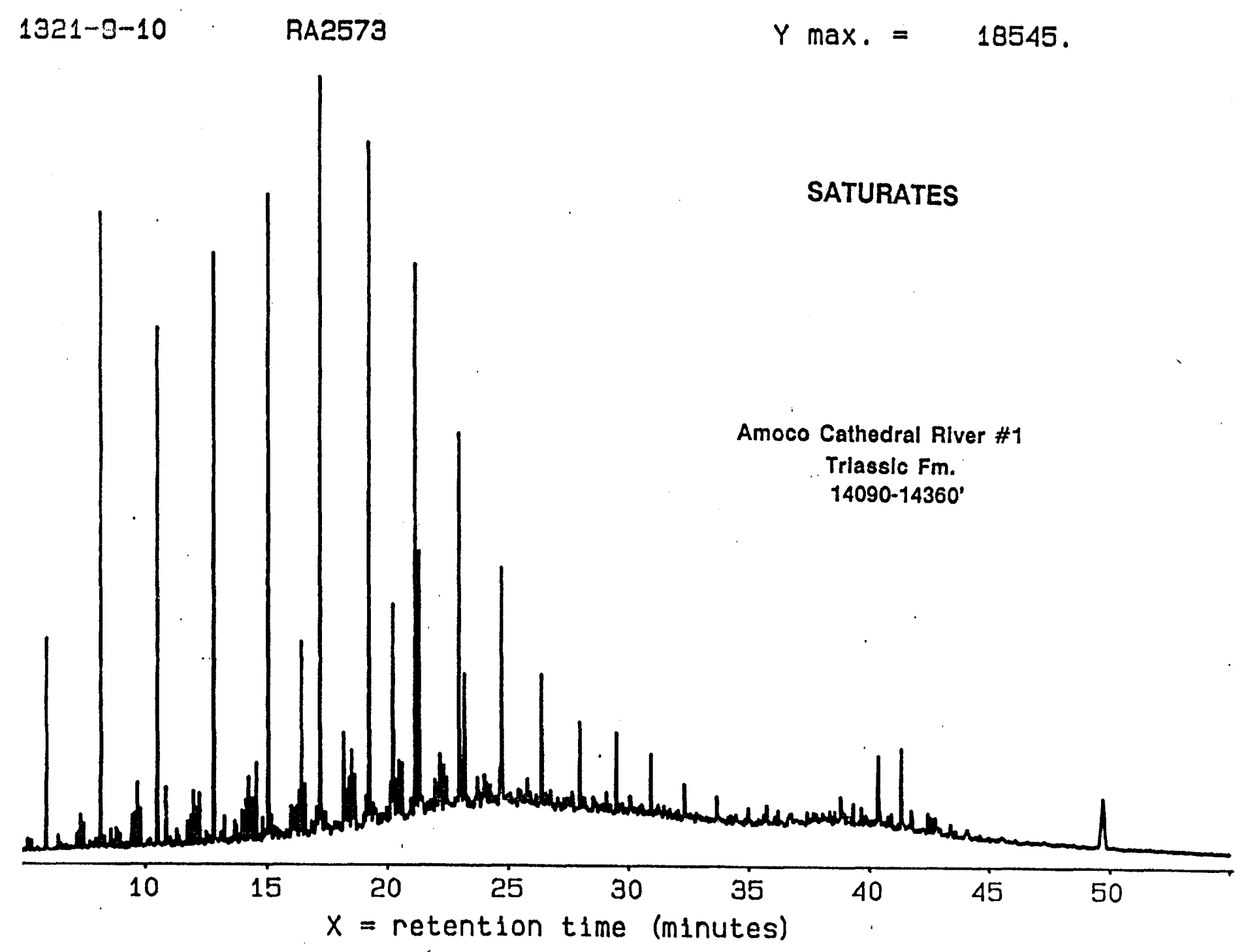

